\title{
Stoichiometric and Non-Stoichiometric Hydrates of Brucine
}

\author{
Doris E. Braun and Ulrich J. Griesser
}

\section{Electronic Supplementary Information}

\section{Contents of Supplementary Information}

1 Crystallographic Information: Cambridge Structural Database (CSD) …............................ 2

2 Determination of Critical Water Activity (Slurry Method) ….............................................. 3

3 Temperature Dependent Slurry Experiments in Water...................................................... 5

4 Variable RH Powder X-ray Diffraction Experiments......................................................... 6

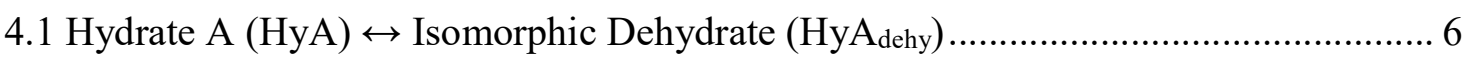

4.2 Hydrate B (HyB) / Amorphous Brucine .................................................................. 8

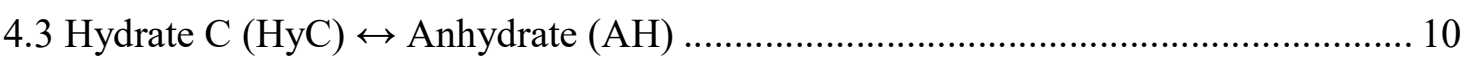

$5 \quad$ Variable Temperature Spectroscopy (IR and Raman) .................................................... 10

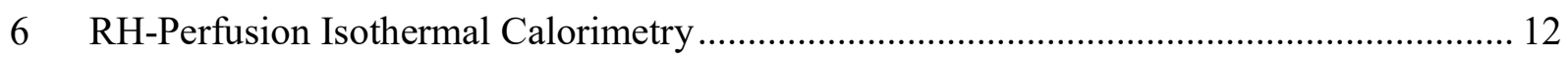

$7 \quad$ 0K Stability Order of Brucine Solid Forms ...................................................................... 13 


\section{Crystallographic Information: Cambridge Structural Database (CSD)}

Table S1. Brucine crystal structures reported in the Cambridge Structural Database (CSD, incl. May 2016 update).

\begin{tabular}{lcc}
\hline CSD-Refcode & Solvate & Ref \\
\hline MAJRIZ & Anhydrate $(\mathbf{A H}$ LT $)$ & 1 \\
MAJRIZ01 & Anhydrate $(\mathbf{A H})$ & 2 \\
CIKDOQ & Dehydrate $(\mathbf{H y A})$ & 3 \\
ZZZPRW01 & Tetrahydrate $(\mathbf{H y B})$ & 4 \\
YOYZIX & 3.86-hydrate $(\mathbf{H y C})$ & 2 \\
DAFFUL & ethanol solvate dihydrate & 5 \\
JIFWEB & dimethanol solvate & 6 \\
MAJRUL & 2-propanol solvate dihydrate & 1 \\
UCOJIG & 5.25 hydrate & 4 \\
JIFWIF & 2-methyl-2-propanol solvate & 6 \\
JIFWOL & 2-methyl-2-butanol solvate & 6 \\
JIFWUR & 3-methyl-2-butanol solvate & 6 \\
PIGNUP & 3,3-dimethyl-2-butanol solvate & 6 \\
PIGPAX & 2,4-dimethyl-3-pentanol solvate & 6 \\
PIGPEB & 1-propanol solvate & 6 \\
PIGPIF & 2-methyl-1-propanol solvate & 6 \\
PIGPOL & 2-methyl-1-butanol solvate & 6 \\
PIGPUR & 3-methyl-1-butanol solvate & 6 \\
PIGQAY & 3-methyl-3-pentanol solvate & 6 \\
HIDGOS & (S)-Butan-1,2-diol solvate hydrate & 7 \\
HIDGUY & (S)-Propane-1,2-diol solvate hydrate & 7 \\
MAJROF & acetone solvate & 1 \\
\hline
\end{tabular}

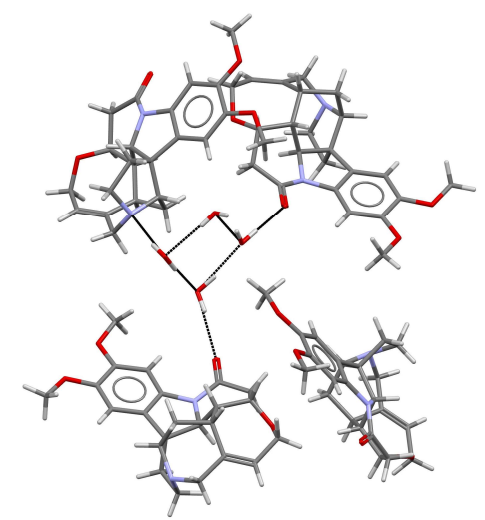

Figure S1. H-bonded intermolecular interactions observed for water molecuels in HyA $\left(\mathrm{CIKDOQ}^{3}\right)$. 


\section{Determination of Critical Water Activity (Slurry Method)}

Excess of amorphous burcine was stirred (500 r.p.m.) in $\geq 0.5 \mathrm{~mL}$ of methanol:water mixtures, each containing a different mole fraction of water corresponding to a defined water activity ${ }^{8,9}$ at $10.0 \pm 0.1{ }^{\circ} \mathrm{C}, 25.0 \pm 0.1{ }^{\circ} \mathrm{C}$ (Figure S2) and $40.0 \pm 0.1{ }^{\circ} \mathrm{C}$ for 7 days. The water activity of solvent mixtures at $10{ }^{\circ} \mathrm{C}$ and $40{ }^{\circ} \mathrm{C}$ was calculated using the NRTL (Non-Random Two-Liquid) model as implemented in the ASPEN Properties software program. ${ }^{10}$ Coulometric Karl Fischer Titration, C20 instrument, Mettler Toledo, $\mathrm{CH}$ ) was applied to determine the water content in the mixtures. Samples were withdrawn, filtered and the resulting phase was determined using PXRD (wet cake measured between two mylar foils to avoid phase transformations).

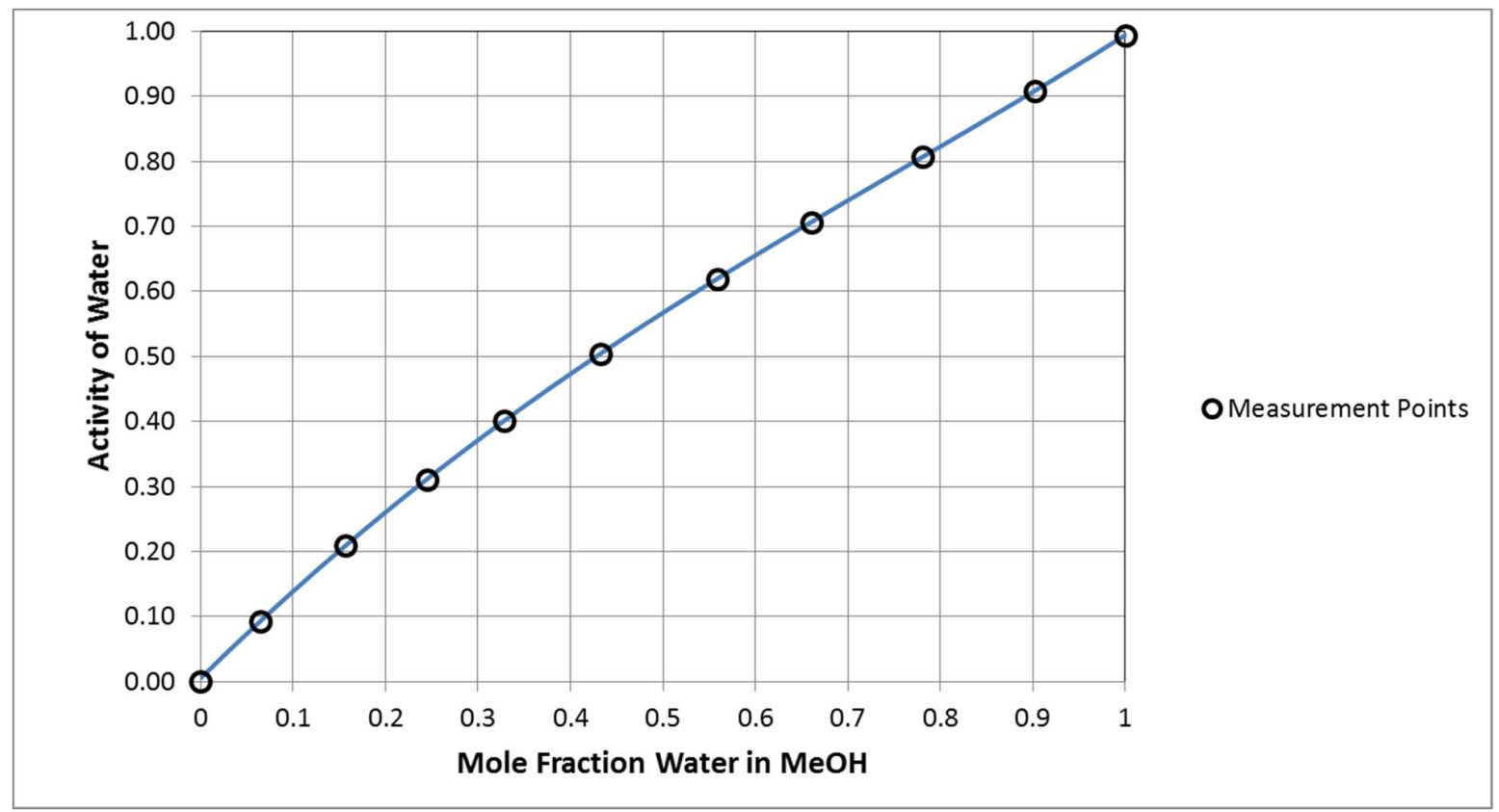

Figure S2. Plot of the water activity versus the mole fraction of water in methanol/water mixtures at $25^{\circ} \mathrm{C}$. Data from refs: ${ }^{8,9}$. 
Table S2. Slurry experiments in $\mathrm{MeOH} /$ water mixtures.

\begin{tabular}{cccccc}
\hline $\begin{array}{c}\mathbf{1 0}^{\circ} \mathbf{C}(\text { Water } \\
\text { Activity) }\end{array}$ & Solid Form & $\begin{array}{c}\mathbf{2 5}^{\circ} \mathbf{C} \text { (Water } \\
\text { Activity) }\end{array}$ & Solid Form & $\begin{array}{c}\mathbf{4 0}^{\circ} \mathbf{C} \text { (Water } \\
\text { Activity) }\end{array}$ & Solid Form \\
\hline$<0.01$ & MeOH-Solvate & $<0.01$ & AH & $<0.01$ & AH \\
0.09 & MeOH-Solvate & 0.09 & AH & 0.09 & AH \\
0.20 & HyA & 0.21 & AH & 0.22 & AH \\
0.30 & HyA & 0.31 & AH & 0.32 & AH \\
0.39 & HyA & 0.40 & HyA & 0.42 & HyA \\
0.49 & HyA & 0.50 & HyA & 0.52 & HyA \\
0.61 & HyA & 0.62 & HyA & 0.63 & HyA \\
0.70 & HyA & 0.71 & HyA & 0.72 & HyA \\
0.80 & HyA & 0.81 & HyA & 0.81 & HyA \\
0.91 & HyB & 0.91 & HyC & 0.91 & HyA \\
1.00 & HyB & 1.00 & HyC & 1.00 & HyA \\
\hline
\end{tabular}




\section{Temperature Dependent Slurry Experiments in Water}

Suspensions of amorphous brucine were prepared in water and then stirred at different temperatures for 7 days. The wet-cakes were analyzed with PXRD (measured between two mylar foils to prevent solvent loss). The results are summarized in Table S3 (constant temperatures) and Table S4 (cycling between $\mathrm{x}$ and $\mathrm{y}^{\circ} \mathrm{C}$ ).

Table S3. Slurry experiments in water (constant temperature).

\begin{tabular}{cc}
\hline Temperature $/{ }^{\circ} \mathbf{C}$ & Solid Form \\
\hline$\sim 2$ & HyB \\
10 & HyB \\
15 & HyB + HyC \\
20 & HyC \\
25 & HyC \\
30 & HyC \\
35 & HyA \\
40 & HyA \\
50 & HyA \\
60 & HyA \\
\hline
\end{tabular}

Table S4. Slurry experiments in water (cycling experiments).

\begin{tabular}{cc}
\hline Temperature $/{ }^{\circ} \mathbf{C}$ & Solid Form \\
\hline $5-10$ & HyB \\
$10-15$ & HyB $>$ HyC \\
$15-20$ & HyB $<$ HyC \\
$20-25$ & $\mathbb{H y C}$ \\
$25-30$ & HyC \\
$30-35$ & $\mathbb{H y A}$ \\
$35-40$ & $\mathbb{H y A}$ \\
$10-20$ & HyB + HyC \\
$20-30$ & $\mathbb{H y C}$ \\
$30-40$ & $\mathbb{H y C}+\mathbb{H y A}$ \\
$40-50$ & $\mathbb{H y A}$ \\
$50-60$ & $\mathbb{H y A}$ \\
\hline
\end{tabular}




\section{Variable RH Powder X-ray Diffraction Experiments}

\subsection{Hydrate A (HyA) $\leftrightarrow$ Isomorphic Dehydrate $\left(\mathrm{Hy}_{\mathrm{A}} \mathbf{A h y}\right)$}

Equilibration conditions (time at respective RH) for Figure 3 a were as follows:

- $40,35,30,25,20,16,14$ and 12\% RH: 4 hours

- $10,8,6$ and $4 \%$ RH: 8 hours

- 2 and $0 \%$ RH: 24 hours

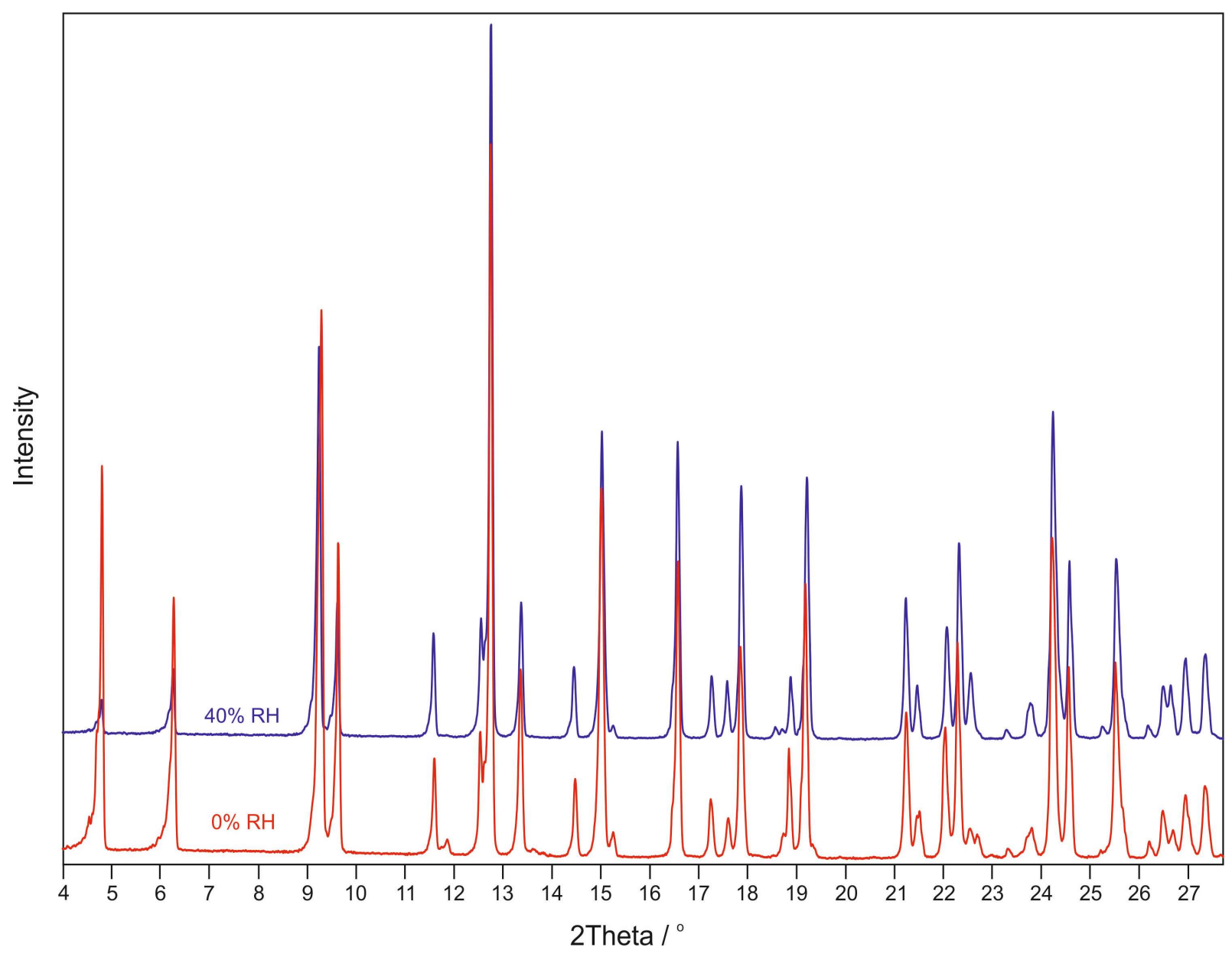

Figure S3. Moisture dependent PXRD measurements of brucine HyA recored at $40 \%$ and $0 \% \mathrm{RH}$. 
Table S5. Refined unit cell parameters, volume, water occupancies $(f)$ and mole ratio dependency on RH for HyA PXRD patterns shown in Figure 3.

\begin{tabular}{|c|c|c|c|c|c|c|c|c|c|c|c|c|}
\hline $\begin{array}{l}\mathbf{T} \\
{ }^{\circ} \mathbf{C}\end{array}$ & $\begin{array}{l}\text { RH } \\
/ \%\end{array}$ & $\begin{array}{l}\mathbf{a} \\
/ \AA\end{array}$ & $\begin{array}{l}\mathbf{b} \\
/ \AA \AA\end{array}$ & $\begin{array}{l}\text { c } \\
/ \AA\end{array}$ & $\begin{array}{l}\beta \\
/ 0\end{array}$ & $\begin{array}{l}\text { Vol } \\
/ \AA^{3}\end{array}$ & $f(\mathrm{~W} 1)$ & $f(\mathrm{~W} 2)$ & $f(\mathbf{W} 3)$ & $f(\mathbf{W} 4)$ & $\begin{array}{l}\text { Mol } \\
\text { ratio } \\
\text { (PXRD) }\end{array}$ & $\begin{array}{l}\text { Mol } \\
\text { ratio } \\
(\text { GMS) }\end{array}$ \\
\hline 25 & 40 & $15.2644(7)$ & $7.4873(3)$ & $19.8770(11)$ & $112.440(4)$ & 2099.72(19) & 1.00 & 1.00 & 1.00 & 1.00 & 2.00 & 2.00 \\
\hline 25 & 35 & $15.2636(7)$ & 7.4874(3) & $19.8768(11)$ & $112.439(4)$ & 2099.62(19) & 0.99 & 1.00 & 1.00 & 1.00 & 2.00 & 1.99 \\
\hline 25 & 30 & $15.2638(7)$ & $7.4876(3)$ & $19.8775(11)$ & $112.437(4)$ & 2099.81(19) & 0.97 & 1.00 & 0.98 & 1.00 & 1.97 & 1.99 \\
\hline 25 & 25 & $15.2635(7)$ & $7.4875(3)$ & $19.8786(11)$ & $112.432(4)$ & 2099.94(18) & 0.96 & 1.00 & 0.94 & 1.00 & 1.95 & 1.98 \\
\hline 25 & 20 & $15.2623(7)$ & $7.4874(3)$ & $19.8784(11)$ & $112.429(4)$ & 2099.76(19) & 0.92 & 1.00 & 0.94 & 1.00 & 1.93 & 1.96 \\
\hline 25 & 16 & $15.2613(7)$ & $7.4877(3)$ & $19.8794(11)$ & $112.423(4)$ & $2099.90(18)$ & 0.89 & 1.00 & 0.93 & 1.00 & 1.91 & 1.93 \\
\hline 25 & 14 & $15.2606(7)$ & 7.4877(3) & $19.8798(11)$ & $112.422(4)$ & 2099.87(19) & 0.89 & 0.97 & 0.93 & 1.00 & 1.89 & 1.89 \\
\hline 25 & 12 & $15.2597(8)$ & $7.4878(3)$ & $19.8797(11)$ & $112.419(4)$ & 2099.78(19) & 0.86 & 0.95 & 0.86 & 1.00 & 1.84 & 1.85 \\
\hline 25 & 10 & $15.2567(8)$ & $7.4875(3)$ & $19.8785(11)$ & $112.420(4)$ & 2099.17(19) & 0.81 & 0.93 & 0.84 & 1.00 & 1.79 & 1.78 \\
\hline 25 & 8 & $15.2546(8)$ & $7.4877(3)$ & $19.8787(12)$ & $112.417(4)$ & $2098.99(20)$ & 0.75 & 0.89 & 0.75 & 1.00 & 1.69 & 1.67 \\
\hline 25 & 6 & $15.2514(8)$ & $7.4878(3)$ & $19.8777(12)$ & $112.419(4)$ & $2098.45(20)$ & 0.65 & 0.76 & 0.68 & 0.92 & 1.50 & 1.50 \\
\hline 25 & 4 & $15.2456(8)$ & $7.4884(3)$ & $19.8687(13)$ & $112.422(4)$ & $2096.83(21)$ & 0.44 & 0.57 & 0.45 & 0.82 & 1.14 & 1.13 \\
\hline 25 & 2 & $15.2415(7)$ & 7.4911(3) & $19.8348(14)$ & $112.346(3)$ & $2094.60(21)$ & 0.07 & 0.23 & 0.17 & 0.44 & 0.46 & 0.59 \\
\hline 25 & 0 & $15.2431(7)$ & $7.4934(3)$ & $19.8091(14)$ & $112.246(3)$ & $2094.23(20)$ & 0.00 & 0.02 & 0.00 & 0.05 & 0.04 & 0.04 \\
\hline
\end{tabular}

${ }^{a}$ GMS - gravimetric moisture sorption/desorption experiments. 


\subsection{Hydrate B (HyB) / Amorphous Brucine}

Equilibration conditions (time at respective $\mathrm{RH}$ ) for Figure $4 \mathrm{~b}$ were as follows:

- $90,85,80,75,70,65,60,55,50,45,40,35,30,25,20,15,10$ and 5\% RH: 6 hours

- $0 \%$ RH: 48 hours

- $5,10,15,20,25,30,35,40,45,50,55,60,65,70,75,80,85,90$ and 95\% RH: 4 hours

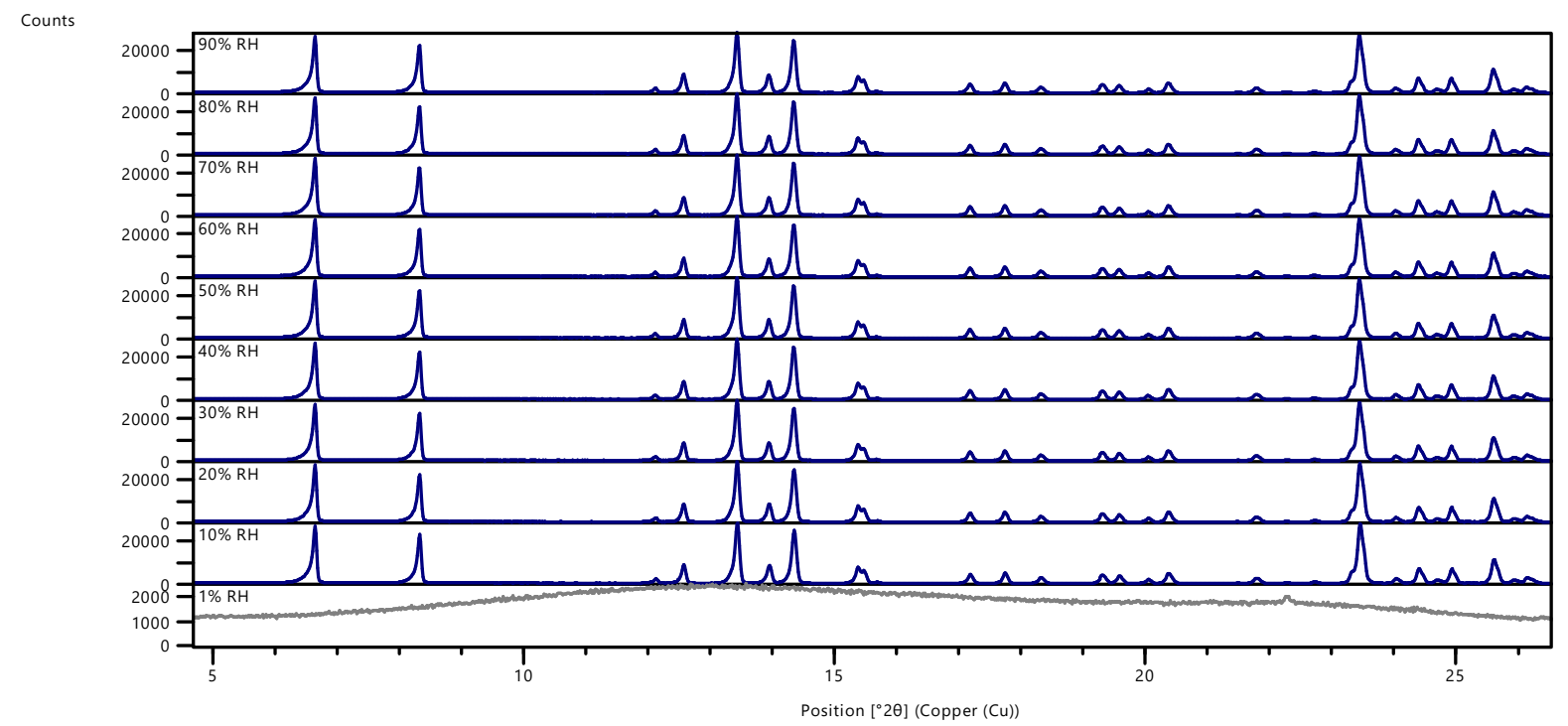

Figure S4. Moisture dependent PXRD measurements of brucine HyB showing the desorption of HyB (top) to amorphous brucine (bottom).

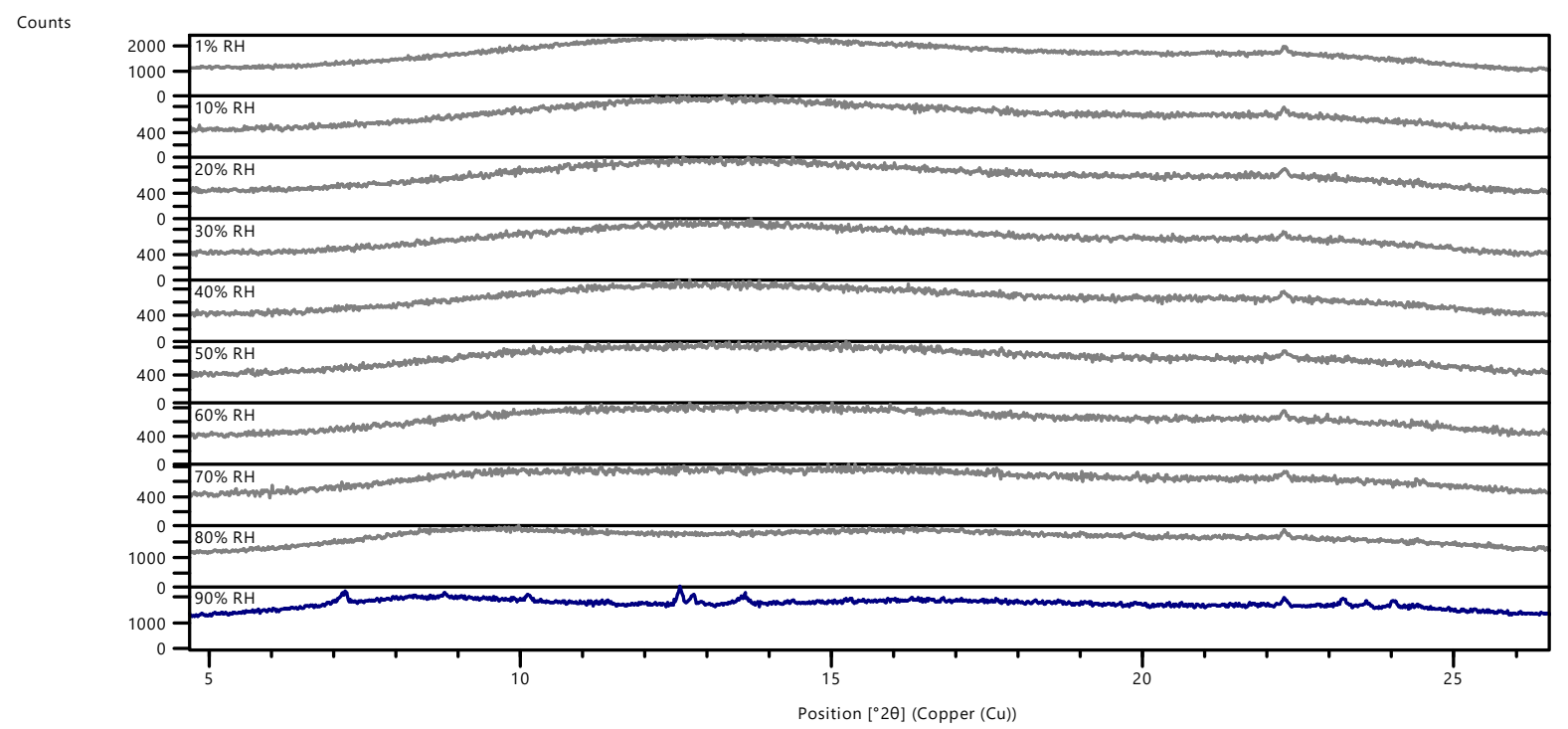

Figure S5. Moisture dependent PXRD measurements of amorphous brucine showing the sorption of amorphous brucine (top) to a mixture of brucine hydrates (bottom). 


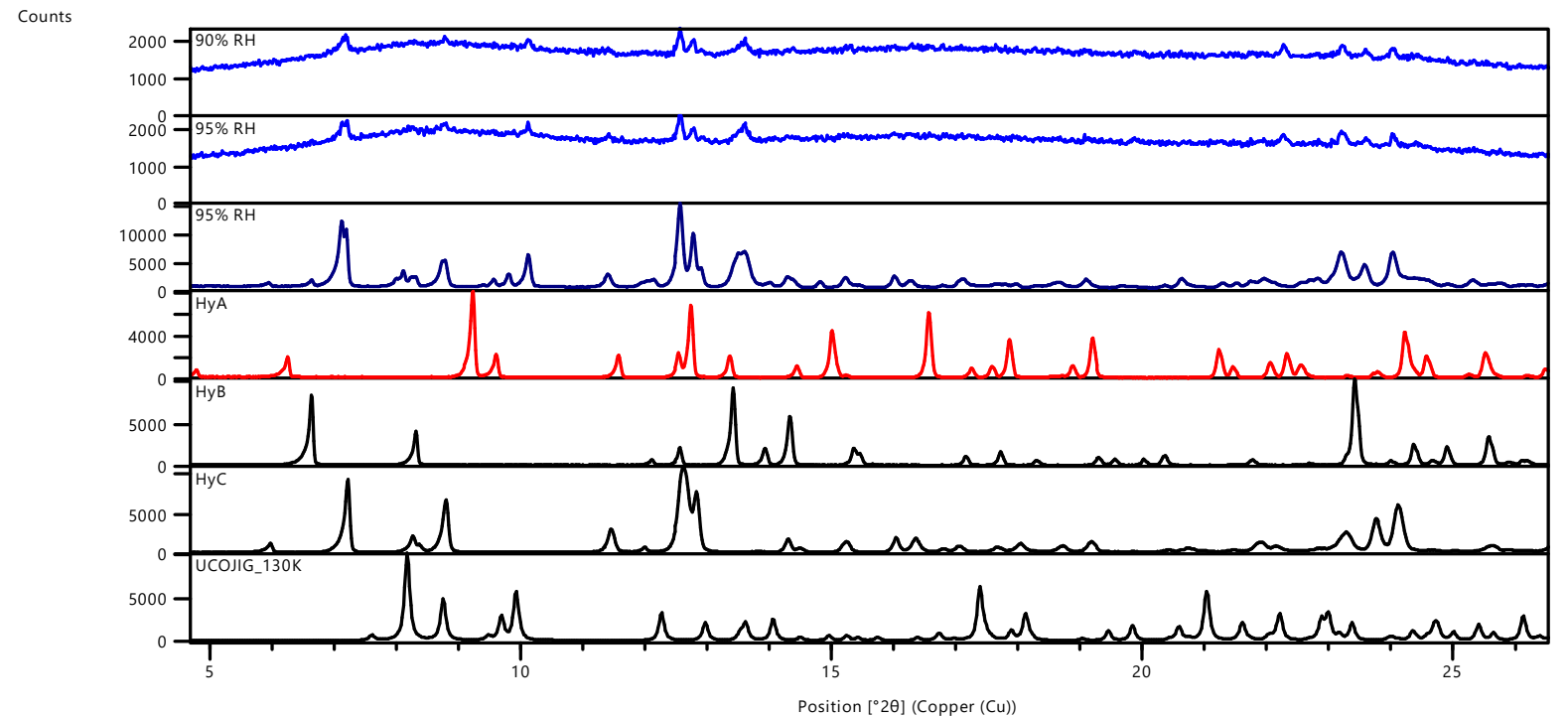

Figure S6. Moisture dependent PXRD measurements of amorphous brucine showing the sorption of amorphous brucine to a mixture of brucine hydrates $(90 \%$ and $95 \% \mathrm{RH})$. The experimental PXRD patterns $(90 \%$ and $95 \% \mathrm{RH})$ are contrasted to reference patterns simulated from the experimental single crystal structures (red and black).

Table S6. Refined unit cell parameters and volume dependency on RH for HyB PXRD patterns.

\begin{tabular}{cccccccccc}
\hline $\mathbf{T} /{ }^{\circ} \mathbf{C}$ & $\mathbf{R H} / \mathbf{\%}$ & $\mathbf{a} / \boldsymbol{\AA}$ & $\mathbf{b} / \boldsymbol{\AA}$ & $\mathbf{c} / \boldsymbol{\AA}$ & $\mathbf{V o l} / \AA^{\mathbf{3}}$ & $\Delta \mathbf{V o l} / \mathbf{\%}$ & $\boldsymbol{R}_{\mathbf{w p}}$ & $\boldsymbol{R}_{\mathbf{e x p}}$ & $\boldsymbol{R}_{\mathbf{p}}$ \\
\hline 25 & 90 & $7.5791(1)$ & $11.5509(2)$ & $26.5359(7)$ & $2323.09(9)$ & 0.00 & 5.27 & 2.59 & 3.85 \\
25 & 80 & $7.5789(1)$ & $11.5502(2)$ & $26.5366(7)$ & $2322.97(9)$ & 0.01 & 5.33 & 2.59 & 3.93 \\
25 & 70 & $7.5784(1)$ & $11.5495(2)$ & $26.5344(7)$ & $2322.48(9)$ & 0.03 & 5.25 & 2.59 & 3.81 \\
25 & 60 & $7.5787(1)$ & $11.5499(2)$ & $26.5350(7)$ & $2322.69(9)$ & 0.02 & 5.35 & 2.61 & 3.89 \\
25 & 50 & $7.5788(1)$ & $11.5496(2)$ & $26.5362(7)$ & $2322.77(9)$ & 0.01 & 5.39 & 2.59 & 3.94 \\
25 & 40 & $7.5785(1)$ & $11.5494(2)$ & $26.5366(7)$ & $2322.66(9)$ & 0.02 & 5.29 & 2.59 & 3.92 \\
25 & 30 & $7.5783(1)$ & $11.5490(2)$ & $26.5360(7)$ & $2322.48(9)$ & 0.03 & 5.27 & 2.59 & 3.87 \\
25 & 20 & $7.5769(1)$ & $11.5472(2)$ & $26.5354(7)$ & $2321.64(9)$ & 0.06 & 5.13 & 2.58 & 3.78 \\
25 & 10 & $7.576(1)$ & $11.5454(2)$ & $26.5370(7)$ & $2321.02(9)$ & 0.09 & 5.20 & 2.56 & 3.84 \\
\hline
\end{tabular}




\subsection{Hydrate C (HyC) $\leftrightarrow$ Anhydrate (AH)}

Equilibration conditions (time at respective $\mathrm{RH}$ ) for Figure $5 \mathrm{~b}$ were as follows:

- $95,90,85,80,75,70,65,60,55,50$ and $45 \%$ RH: 4 hours

- $40 \%$ RH: 8 hours

- $35,30,25,20,15,10,5,0 \%: 4$ hours

- $5,10,15,20,25,30,35,40,45,50,55,60,65$ and 70\% RH: 2 hours

- 75 and 80\% RH: 4 hours

- $\quad 85,90$ and 95\% RH: 24 hours

Table S7. Refined unit cell parameters and volume dependency on RH for HyC PXRD patterns.

\begin{tabular}{|c|c|c|c|c|c|c|c|c|c|c|}
\hline $\begin{array}{c}\mathbf{T} \\
{ }^{\circ} \mathbf{C}\end{array}$ & $\begin{array}{l}\text { RH } \\
/ \%\end{array}$ & a $/ \AA ̊$ & $\mathbf{b} / \AA$ & c /Å & $\beta / /^{\circ}$ & Vol / $\AA^{3}$ & $\begin{array}{c}\Delta \mathrm{Vol} \\
/ \%\end{array}$ & $R_{\mathrm{wp}}$ & $R_{\exp }$ & $R_{\mathrm{p}}$ \\
\hline 25 & 95 & $25.0257(20)$ & $12.3817(7)$ & $17.4716(11)$ & $122.217(3)$ & $4580.25(55)$ & & 5.86 & & 4.20 \\
\hline 25 & 90 & & 3819 & & ) & & & 0 & & 4.04 \\
\hline 25 & 80 & & 12.38 & & $2.204(2)$ & $1(31)$ & & 9 & & 3.67 \\
\hline 25 & 70 & $25.0150(11)$ & $12.3806(4)$ & & $122.219(2)$ & $4573.70(32)$ & & & & 3.65 \\
\hline 25 & 60 & & $12.3787(4)$ & & $122.237(2)$ & $4570.77(32)$ & & 5.18 & 2.53 & 3.72 \\
\hline 25 & 55 & $25.0131(13)$ & $12.3790(5)$ & $17.4535(7)$ & $122.247(2)$ & $4570.68(36)$ & 0.21 & 5.73 & 2.53 & 4.02 \\
\hline
\end{tabular}

\section{Variable Temperature Spectroscopy (IR and Raman)}

Infrared Spectra were recorded with a Bruker Vertex 70 spectrometer (Bruker Analytische Messtechnik GmbH, D). Potassium bromide $(\mathrm{KBr})$ discs $(13 \mathrm{~mm})$ were prepared by gently grinding and mixing $1-2 \mathrm{mg}$ sample with $230 \mathrm{mg} \mathrm{KBr}$ in a mortar with pestle, evacuating of the mixture in the pressing tool for 20 seconds at $\sim 10$ mbar and applying a pressure of about $800 \mathrm{MPa}$ for about 60 seconds using a hydraulic press. The spectra were recorded in the range of 4000 to $400 \mathrm{~cm}^{-1}$ at an instrument resolution of $2 \mathrm{~cm}^{-1}$ (64 scans per spectrum).

Raman spectra were recorded with a Bruker RFS 100 Raman-spectrometer (Bruker Analytische Messtechnik GmbH, D), equipped with a Nd:YAG Laser (1064 nm) as the excitation source and a liquid-nitrogen-cooled, high sensitivity Ge-detector. The spectra (64 scans per spectrum) were recorded in aluminum sample holders with a laser power of $300 \mathrm{~mW}$ and a resolution of $2 \mathrm{~cm}^{-1}$.

Temperature conditions, for both IR and Raman measurements, were adjusted with a SPECAC (Grasebury Specac Limited, Orpington, UK) variable temperature cell and a temperature control unit. 
Raman and IR spectra were recorded for HyA every $2{ }^{\circ} \mathrm{C}$ from 25 to $161{ }^{\circ} \mathrm{C}$ (Figures S7 and S8) to follow the thermal events observed in the DSC experiments. Ignoring thermal effects, three distinct phases can be identified during the dehydration process in Figure S7: HyA (blue), amorphous brucine (green), and AH (red). Small variations within the HyA Raman spectra indicate the variable water content between 25 and $85^{\circ} \mathrm{C}$. In agreement, the variable temperature IR spectra show hardly any changes, apart from a significant decrease in the intensity of the $v(\mathrm{O}-$ H) bands, below $90{ }^{\circ} \mathrm{C}$ (Figure S8).
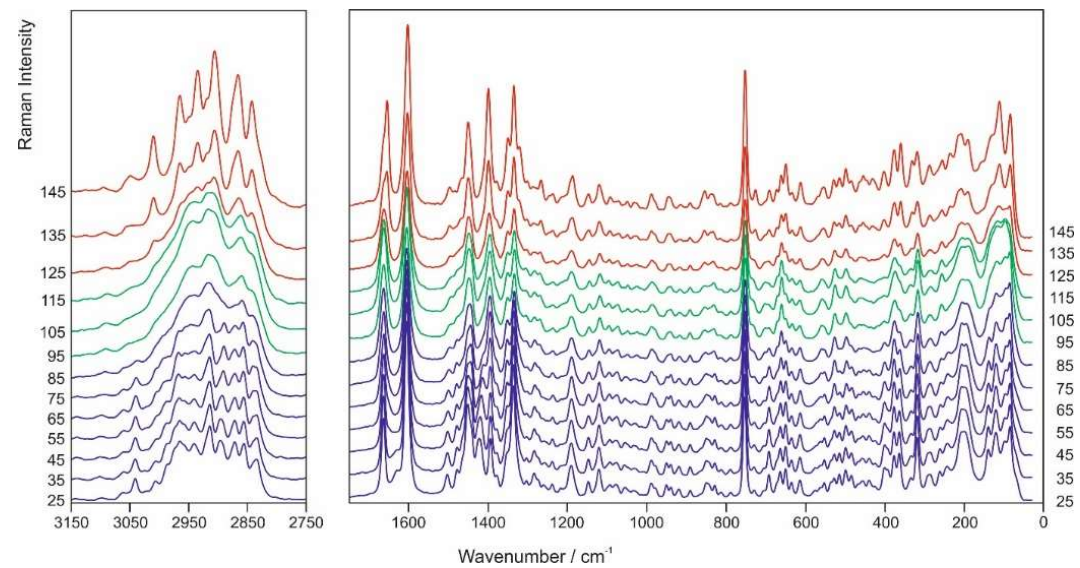

Figure S7. FT-Raman spectra of brucine HyA recorded at temperatures ranging from 25 to 145 ${ }^{\circ} \mathrm{C}$. Numbers on the $\mathrm{y}$-axis indicate the measurement temperature in ${ }^{\circ} \mathrm{C}$.

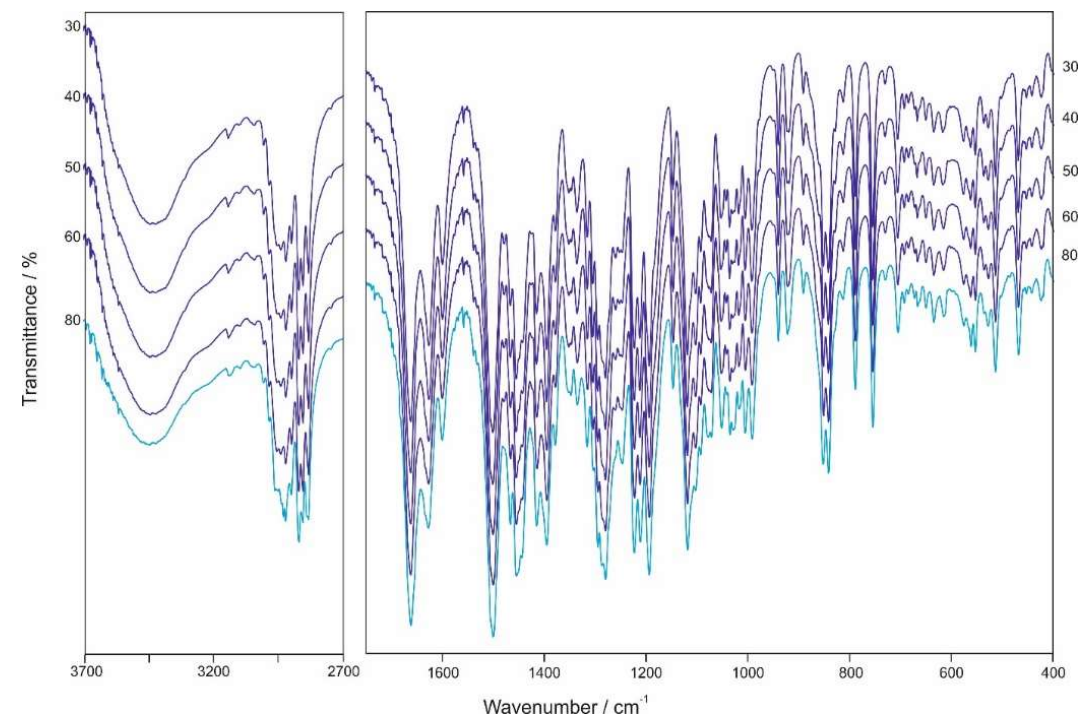

Figure S8. Selection of FT-IR spectra of brucine HyA recorded at temperatures ranging from 25 to $80{ }^{\circ} \mathrm{C}$. Numbers on the y-axis indicate the measurement temperature in ${ }^{\circ} \mathrm{C}$. 


\section{RH-Perfusion Isothermal Calorimetry}

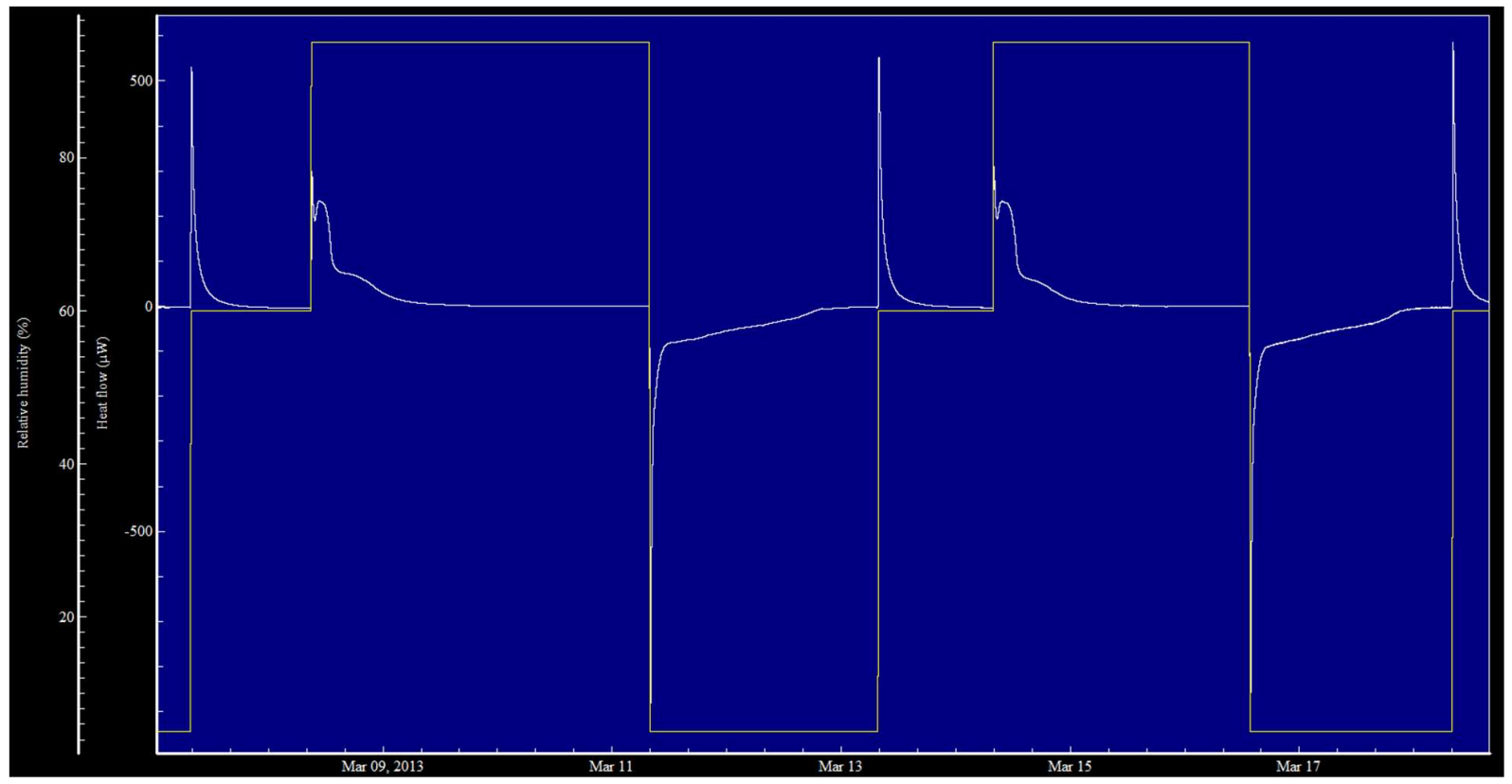

Figure S9. Isothermal RH-Perfusion calorimetry showing the hydration/dehydration reactions of brucine $\mathbf{A H} / \mathbf{H y C}$.

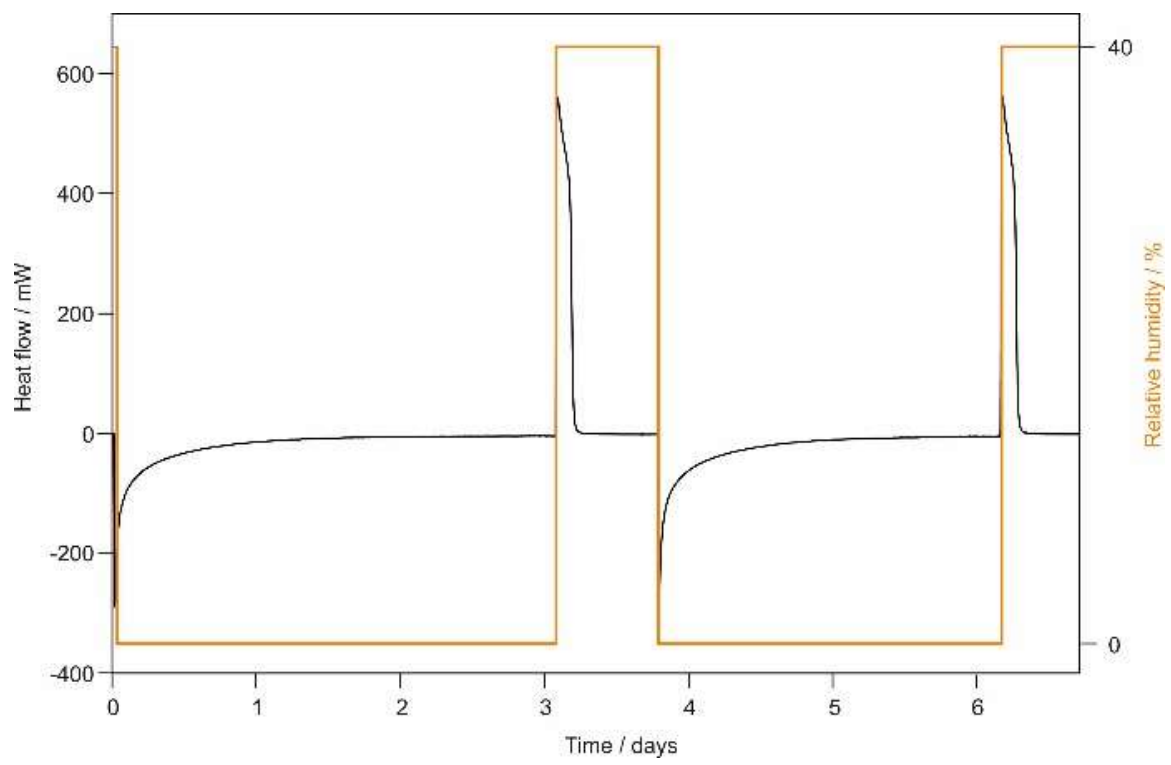

Figure S10. Isothermal RH-Perfusion calorimetry showing the dehydration/hydration reactions of brucine $\mathbf{H y A} / \mathbf{H y A}_{\text {dehy. }}$ 
7 0K Stability Order of Brucine Solid Forms

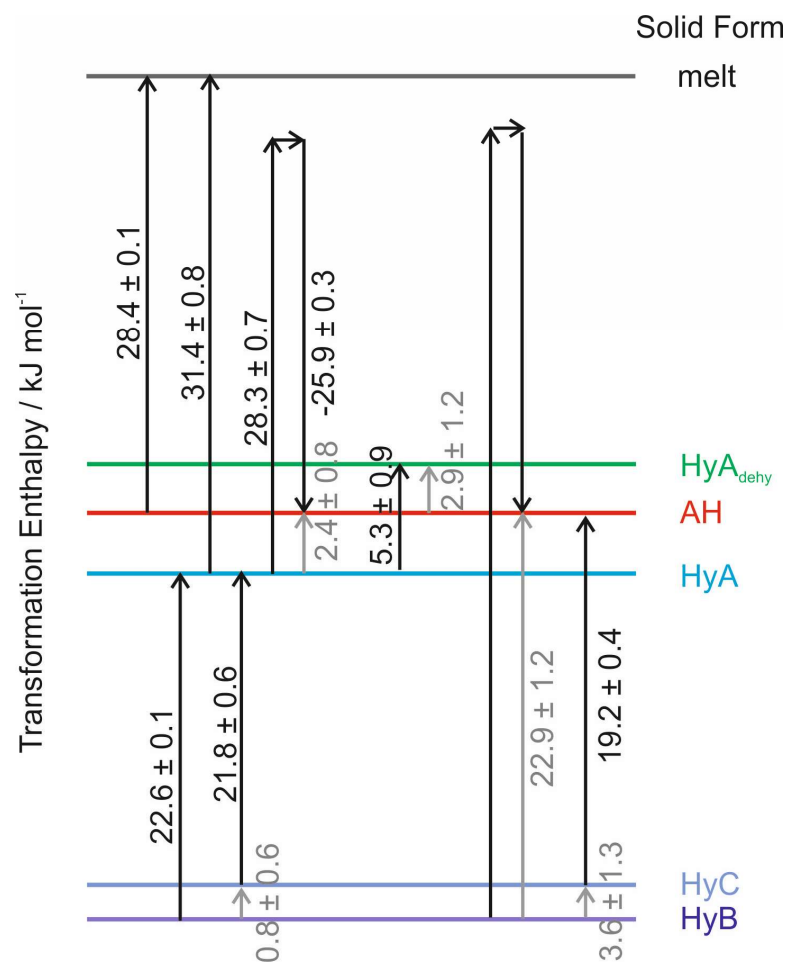

Figure S11. Overview of experimentally determined heat of transformations. Numbers correspond to $\Delta_{\mathrm{trs}} H$ in $\mathrm{kJ} \mathrm{mol}^{-1}$ (as given in Table 2). Horizontal lines indicate the different brucine solid forms. 0K stability order is as follows: HyB (most stable) $>\mathbf{H y C}>\mathbf{H y A}>$ $\mathbf{A H}>\mathbf{H y} \mathbf{A}_{\text {dehy. }}$. 


\section{Reference List}

1. Bialonska, A.; Ciunik, Z. Acta Crystallogr., Sect. C: Cryst. Struct. Commun. 2004, C60, o853-o855.

2. Bialonska, A.; Ciunik, Z.; Ilczyszyn, M. M.; Siczek, M. Cryst. Growth Des. 2014, 14, 6537-6541.

3. Smith, G.; Wermuth, U. D.; White, J. M. Acta Crystallogr. C 2007, 63, o489-o492.

4. Smith, G.; Wermuth, U. D.; Healy, P. C.; White, J. M. Acta Crystallogr., Sect. C: Cryst. Struct. Commun. 2006, C62, o203-o207.

5. Glover, S. S. B.; Gould, R. O.; Walkinshaw, M. D. Acta Crystallogr. , Sect. C: Cryst. Struct. Commun. 1985, C41, 990-994.

6. Watabe, T.; Kobayashi, K.; Hisaki, I.; Tohnai, N.; Miyata, M. Bull. Chem. Soc. Jpn. 2007, 80, 464-475.

7. Bialonska, A.; Ciunik, Z. CrystEngComm 2013, 15, 5681-5687.

8. Goelles, F. Monatsh. Chem. 1961, 92, 981-991.

9. Zhu, H.; Yuen, C.; Grant, D. J. W. Int. J. Pharm. 1996, 135, 151-160.

10. Aspen Properties, version 8.4; Aspen Technology: 2015 